\title{
INCIDENCIA DEL CONTROL EN LA EFICIENCIA DE COMPRAS DIRECTAS EN LA ZONA REGISTRAL N XIII - SEDE TACNA. 2016
}

\author{
INCIDENCE OF CONTROL IN THE EFFICIENCY OF DIRECT PURCHASES \\ IN REGISTRATION AREA No. XIII - TACNA SITE. 2016
}

\section{Resumen}

La investigación tiene por objetivo determinar la incidencia del nivel de control en la eficiencia de compras directas en la Zona Registral $N^{\circ}$ XIII Sede Tacna, 2016. Se afirma que el nivel de control incide significativamente en la eficiencia de compras directas en la Zona Registral $N^{\circ} \mathrm{XIII-}$ Sede Tacna, 2016. La investigación se desarrolló bajo un enfoque cuantitativo, investigación no experimental o "ex post facto" y correlacional porque no se manipulan las variables y más bien por análisis se busca establecer las relaciones causa - efecto y correlación de las variables $\mathrm{X} e$ Y. Se aplicaron dos instrumentos a toda la muestra, para medir las variables: Nivel de eficiencia de compras directas y para medir la variable nivel de control; las cuales permitieron recoger información, relacionar y medir las variables de estudio, efectuar las correlaciones y comparaciones correspondientes. La muestra estuvo conformada por 81 personas relacionadas con la Zona Registral $\mathrm{N}^{\circ} \mathrm{XIII}$ - Sede Tacna. Los resultados obtenidos permitieron establecer que: El nivel de control incide significativamente en la eficiencia de compras directas en la Zona Registral N XIII - Sede Tacna. 2016.

Palabras Clave: Eficiencia, nivel de control
FREDDY SILVERIO COPA COLQUE 1

\section{Abstract}

The objective of the research is to determine the effect of the level of control on the efficiency of direct purchases in the Registration Zone No. XIII - Tacna Headquarters. 2016. It is affirmed that the level of control has a significant impact on the efficiency of direct purchases in the Registration Zone No. XIII - Sede Tacna, 2016. The research was developed under a quantitative approach, non-experimental or "ex post facto" and correlational research because the variables are not manipulated and rather by analysis we try to establish the cause - effect relationships and correlation of the variables $X$ and $Y$. Two instruments were applied to the whole sample, to measure the variables: Efficiency level of direct purchases and for measure the variable level of control; which allowed us to collect information, relate and measure the study variables, make correlations and comparisons. The sample consisted of 81 people related to the Registration Zone No. XIII - Tacna Headquarters. The results obtained allowed us to establish that: The level of control has a significant impact on the efficiency of direct purchases in the Registration Zone No. XIII - Tacna Headquarters. 2016.

Keywords: Efficiency, level of control.

\section{INTRODUCCIÓN}

En la actualidad los procesos de compras están calificados de poco transparentes, a pesar que se han dado algunos avances con la Ley de Transparencia, cuya instalación ha costado tanto esfuerzo, pero aún no se ve los resultados de eficiencia en el tema de las compras estatales. Esta investigación busca que el Organismo Supervisor de las Contrataciones del Estado
(Organismo Supervisor de las Contrataciones del Estado, 2013), implemente procedimientos para la ejecución de las contrataciones de bienes y servicios iguales o menores a ocho unidades impositivas tributarias (UIT-s), al no estar dentro de los alcances de la Ley de Contrataciones del Estado. En la actualidad muchas de las entidades que se rigen por la Ley de Contrataciones del

1 MAESTRO EN GESTIÓN Y POLÍTICAS PÚBLICAS 
Estado, tienen que elaborar su propio procedimiento para poder controlar las contrataciones (compras directas) cuyos montos sean iguales o menores a ocho Unidades Impositivas Tributarias (8 UIT), al momento de su transacción, dado que están fuera de la aplicación de dicha Ley.

Según Gómez (2013), las reformas de la contabilidad gubernamental en Colombia y en Perú, son innovaciones en gobernanza. Los casos de los departamentos de Colombia y Perú, nos permiten identificar que las reformas de la gestión financiera pública están impactando en la transparencia". Según Ronald (2011), deja sentada la importante función de los sistemas de información en todas las áreas del conocimiento y en todas las funciones que puedan desarrollarse en una institución, así como la relación que tiene y los aportes que otorga en problemas de índole social y ética. Paima (2013) al realizar el análisis de la gestión en el área de compras de la empresa comercializadora de vehículos Autonort Trujillo S.A, se tuvo en consideración los procedimientos empleados por los encargados del control y gestión de las compras en dicha área; considerando las condiciones de calidad y oportunidad, la provisión de bienes o servicios que se haga al mínimo costo con la máxima eficiencia y el mejor uso de los recursos económicos disponibles. Caroy (2010) análisis del efecto en la generación de empleo, de la política de fomento de la pequeña empresa a través de las compras estatales en el Perú, afirma que el diseño de la política del Estado estaba orientado a favorecer más a la microempresa, a fin de tener mayor impacto sobre el empleo y la calidad del mismo. Avilés (2007) afirma que el fraccionamiento es la modalidad de corrupción con más frecuencia en las adquisiciones que se utiliza para evitar procesos más complejos y transparentes, buscándose adquirir a través de procesos pequeños y discretos. Cruz (2011) concluye que el Sistema Nacional de Control Interno Gubernamental en la gestión de la Unidad de Logística representa una brecha promedio integrada de $72.22 \%$, debido fundamentalmente a que los funcionarios y empleados de la entidad desconocían o aplicaron mal las normas de control gubernamental. Zarpan (2013) en la Municipalidad Distrital de Pomalca -2012, identifica cinco riesgos potenciales: adquisición de productos innecesarios; excesivos costos de bienes adquiridos, extravió, deterioro, robo sistemático de los bienes almacenados; deficiente desempeño del recurso humano.
Torricelli (2006), concluye que uno de los efectos positivos es que, la nueva ley de contrataciones y adquisiciones del Estado reduce los trámites porque permite a los usuarios simplificar y ser más ágiles en sus adquisiciones con el Estado.

"El control gubernamental, consiste en la supervisión, vigilancia y verificación de los actos y resultados de la gestión pública...." (Caroy, 2010).En el país, el control gubernamental se encuentra normado en la Ley Orgánica del Sistema Nacional de Control y de la Contraloría General de la República - Ley N²7785. Esta Ley orgánica del Sistema Nacional de Control y de la Contraloría General de la República tiene como objetivo propender al apropiado, oportuno y efectivo ejercicio del control gubernamental, para prevenir y verificar, mediante la aplicación de principios, sistemas y procedimientos técnicos, la correcta, eficiente y transparente utilización y gestión de los recursos y bienes del Estado". (Caroy, 2010, pág. 74). Los citados principios son de observancia obligatoria por los órganos de control y pueden ser ampliados o modificados por la Contraloría General, a quien compete su interpretación. (Alvarado, 2008, pág. 103). "La Contraloría General organiza y desarrolla el control gubernamental en forma descentralizada y permanente......." (Gómez, 2013, pág. 71).

El Control interno del área de compras es el plan de organización entre el sistema de contabilidad, funciones de empleados y procedimientos coordinados que tienen por objeto obtener información segura, oportuna y confiable, así como promover la eficiencia de operaciones de adquisición o compra" (Singaucho, 2010). Se considera al conjunto de planes, métodos y procedimientos necesarios para garantizar que las actividades de la entidad se realicen de conformidad con la ley vigente. El control interno es efectuado por diversos niveles jerárquicos. Los funcionarios, auditores internos y personal de menor nivel contribuyen para que el sistema de control interno funcione con eficacia, eficiencia y economía (Avilés, 2007). El titular o funcionario designado de cada entidad debe identificar los riesgos que implican las operaciones y, estimar sus márgenes aceptables en términos cuantitativos y cualitativos, de acuerdo con las circunstancias (Suárez, 2011). Sin embargo una estructura de control interno no puede garantizar por sí misma una gestión eficaz y eficiente(Paima \& Villalobos, 2013).

La Ley de Contrataciones del Estado es un instrumento que permite a las diferentes entidades del Estado realizar contratos de bienes 
y servicios y para la ejecución de obras". (Caballero, L., 2011). Calimeri (2002) manifiesta que la gestión de compras se realiza tomando en cuenta las necesidades de adquirir las materias estrictamente necesarias, las cantidades a comprar acorde con el plan de producción, estudiar la calidad de los materiales, examinar la base de proveedores y negociar los más altos plazos de crédito. Se conoce que el departamento de compras maneja junto con el almacén los llamados stocks mínimos donde el inventario juega un rol importante (Schultz ,1998).
Las alianzas hoy en día son la fuente de aprendizaje y diferencia competitiva. Thompson y Strickland III (2000), dicen: “... las alianzas pueden incluir esfuerzos comunes de investigación, intercambio de tecnología, uso conjunto de las instalaciones de producción, venta de productos entre sí". Velázquez (2002), conceptualiza las Alianzas Estratégicas como una unión voluntaria, duradera y organizada de personas y/o empresas, que ponen sus fuerzas en común para lograr ventajas especiales que les permitan una mejor situación competitiva.

\section{OBJETIVOS}

a) Conocer el nivel de control en las compras cuyos montos son iguales o menores a ocho (8) Unidades Impositivas Tributaria en la Zona Registral $N^{\circ}$ XIII - Sede Tacna. b) Medir el nivel de eficiencia que alcanza las compras directas en la Zona Registral $\mathrm{N}^{\circ} \mathrm{XIII}$ Sede Tacna.

\section{METODOLOGÍA}

Estudio transeccional - no experimental. El trabajo se aplicó a la SUNARP - Zona Registral $N^{\circ}$ XIII - Sede Tacna, el cual comprende el primer semestre del 2016. Las unidades de esta investigación serán los operadores logísticos y personal SUNARP de la Zona Registral $N^{\circ}$ XIII -
Sede Tacna, mediante una muestra representativa de 81 personas. El instrumento de medición aplicado fue sometida a la prueba de validez (juicio de expertos) y confiabilidad (Alfa de Cronbach de 0,82 ).

\section{RESULTADOS}

El $43 \%$ de los encuestados manifiestan que "a veces" en las compras directas se espera de quienes participan sean funcionarios públicos $o$ proveedores - un comportamiento leal y respetuoso de las normas, el $42 \%$ manifiestan que "a veces" en las compras directas se garantiza que ofrezca oportunidades reales y justas de participación, el $47 \%$ manifiestan que "a veces" en las compras directas se informa la fecha de su registro y el estado en que se encuentra dicha contratación, el 37\% que "casi siempre" en las compras directas la información contiene el monto total de la contratación efectuada, independientemente de si agrupa diferentes ítems, el $42 \%$ que "casi nunca" en las compras directas la celebración de los contratos sigue procedimientos obligatorios establecidos por normas constitucionales, legislativas y reglamentarias, el $42 \%$ que "casi nunca" la entidad cuenta con directiva que norme el procedimiento para las contrataciones por montos iguales o menores a tres (8) UIT-s, el 43\% que "a veces" los criterios para determinar cuál es el proceso de selección a seguir en cada caso, son varios que actúan complementariamente entre sí, el $47 \%$ que "a veces" la normativa que regula el procedimiento para las compras directas carece de supervisión por parte del Órgano de Control Institucional $(\mathrm{OCl})$, dado que no existe ningún procedimiento que ellos puedan aplicar, el $39 \%$ que "casi nunca" se ha establecido la obligación de las entidades de aprobar, publicar y comunicar su Plan Anual de Contrataciones, y, sólo el $2 \%$ manifiesta lo contrario el $46 \%$ manifiestan que "casi nunca" se ha establecido un instrumento de gestión para la entidad como fuente de información para los proveedores acerca de las necesidades de las entidades en materia de contratación, el $41 \%$ manifiestan que "casi nunca" las compras directas facilitan la administración pública el cumplimiento de la Normativa de Contrataciones 
y Adquisiciones del Estado, el 37\% que "casi nunca" las compras directas reducen el número de procesos competitivos, el $44 \%$ que "casi nunca" en las compras directas se tiene elaborado su propio procedimiento con el fin de garantizar que se efectúen las compras directas en condiciones de eficacia, el $43 \%$ que "casi nunca" en las compras directas se contempla la posibilidad de utilizar medios electrónicos en la contratación con el estado, el $43 \%$ que "casi nunca" las compras directas permiten a la administración pública comprar de forma eficiente, económica y oportuna para satisfacer adecuadamente las necesidades y metas trazadas.

Asimismo, respecto a la evaluación del nivel de control en las compras directas, el $41 \%$ manifiestan que "casi nunca" la entidad cuenta con normas que regulen el procedimiento para las contrataciones por montos iguales o menores a ocho (8) UIT-s., el $42 \%$ que "casi nunca" el Jefe del Órgano de Control Institucional, realiza alguna acción de control a las contrataciones por montos iguales o menores a ocho (8) UIT-s, el $38 \%$ que "casi nunca" el área de Logística aplica penalidades a las órdenes de compra o de servicio iguales o menores a ocho (8) UIT-s por incumplimiento en el plazo establecido; el $43 \%$ que "a veces" se realizan adecuados procedimientos en las contrataciones por montos iguales o menores a ocho (8) UIT-s; el $46 \%$ que "a veces" se adoptan normas para la protección de los recursos en el marco del proceso de control; el
$41 \%$ de los encuestados manifiestan que "casi nunca" se realiza supervisión en contrataciones por montos iguales o menores a ocho (8 UIT-s;

Respecto al Nivel de riesgo en las compras directas, el $28 \%$ manifiestan que "casi siempre" la exclusión en la Ley a las contrataciones directas por montos iguales o menores a ocho unidades impositivas tributarias (8) UIT-s, pone en riesgo la transparencia de estas contrataciones; el $38 \%$ que "casi nunca" los procedimientos en las contrataciones por montos iguales o menores a ocho (8) UIT-s, garantizan su eficiencia.; el $28 \%$ que "casi siempre" la entidad carece de supervisión por parte del Órgano de Control Institucional $(\mathrm{OCl})$, en cuanto a las compras directas dado que no existe ningún procedimiento que ellos puedan aplicar; el $31 \%$ que "casi siempre" el OSCE, al excluir de la Ley a las contrataciones directas, genera suspicacias en el manejo de los recursos al no existir ningún procedimiento general que regule este tipo de contrataciones dando lugar a incurrir en corrupción; y el $28 \%$ que "casi siempre" la ausencia de implementación de normatividad en las contrataciones directas incide en el nivel de riesgo de estas.

En la figura 1 , se observa que el $43 \%$ refieren que el nivel de transparencia en el control de las compras directas es bajo. Asimismo, el $37 \%$ de los encuestados señalan que alcanzan un nivel medio; y el $20 \%$ de los encuestados manifiestan que es alto.

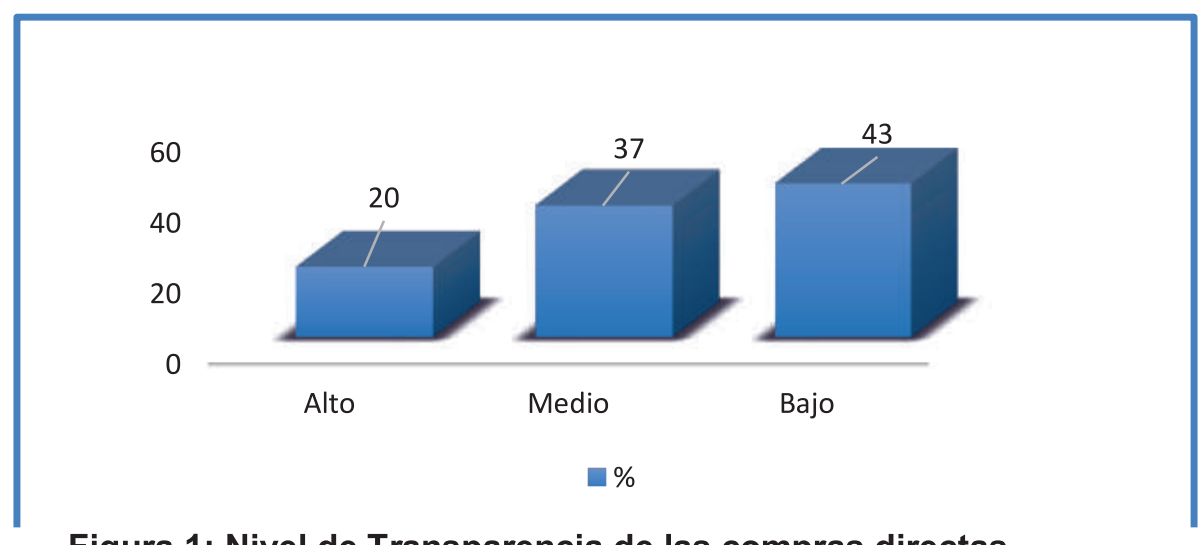

Figura 1: Nivel de Transparencia de las compras directas

Asimismo, el $52 \%$ refieren que el nivel de manejo administrativo en el control de las compras directas es bajo. Asimismo, el $32 \%$ de los encuestados señalan que alcanzan un nivel medio; y el $16 \%$ de los encuestados manifiestan que es alto (Fig. 2) 


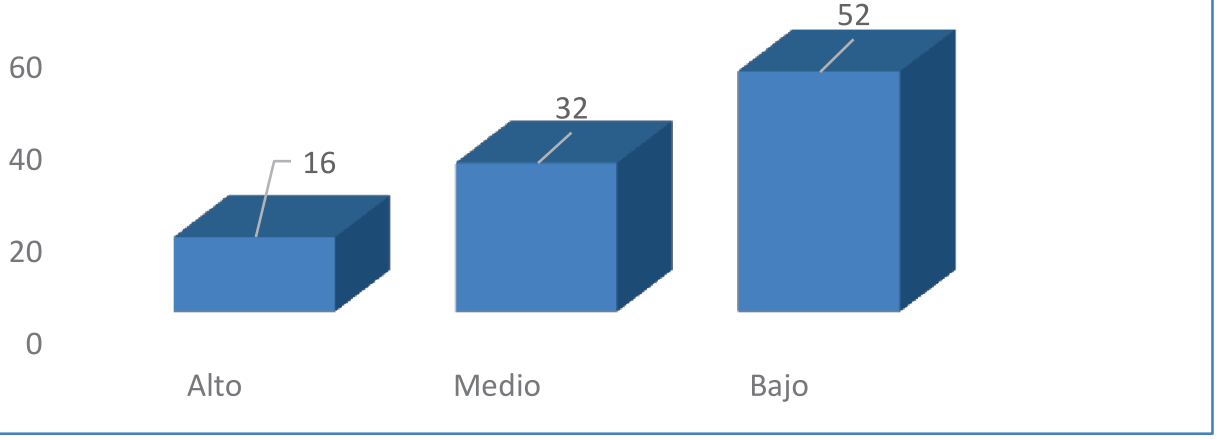

Figura 2: Nivel de manejo administrativo de las compras directas.

El $67 \%$ refieren que el nivel de manejo de eficacia en el control de las compras directas es bajo. El $57 \%$ refieren que el nivel de normatividad de las compras directas también es bajo asi como que el $62 \%$ refieren que el nivel de supervisión que alcanzan las compras directas es en ese mismo nivel no adecuado. El $40 \%$ refieren que el nivel de riesgo que alcanzan las compras directas es medio.

Dada una muestra de 81 observaciones con una media de 2.77 y una desviación estándar de 0.6, el estadístico Z calculado es igual a 49.1833, la cota de confianza muestra que los valores de mas soportados por los datos son mayores o iguales que 2.69408 ( $p<0.05)$ (Fig.3). El nivel de control en las compras cuyos montos son iguales o menores a ocho (8) Unidades Impositivas Tributaria en la Zona Registral $N^{\circ}$ XIII - Sede Tacna, es bajo.

Tambien se cfomprueba que el nivel de eficiencia que alcanza las compras directas en la Zona Registral $N^{\circ}$ XIl es bajo ( $\left.p:<0.05\right)$ Fig. 03.

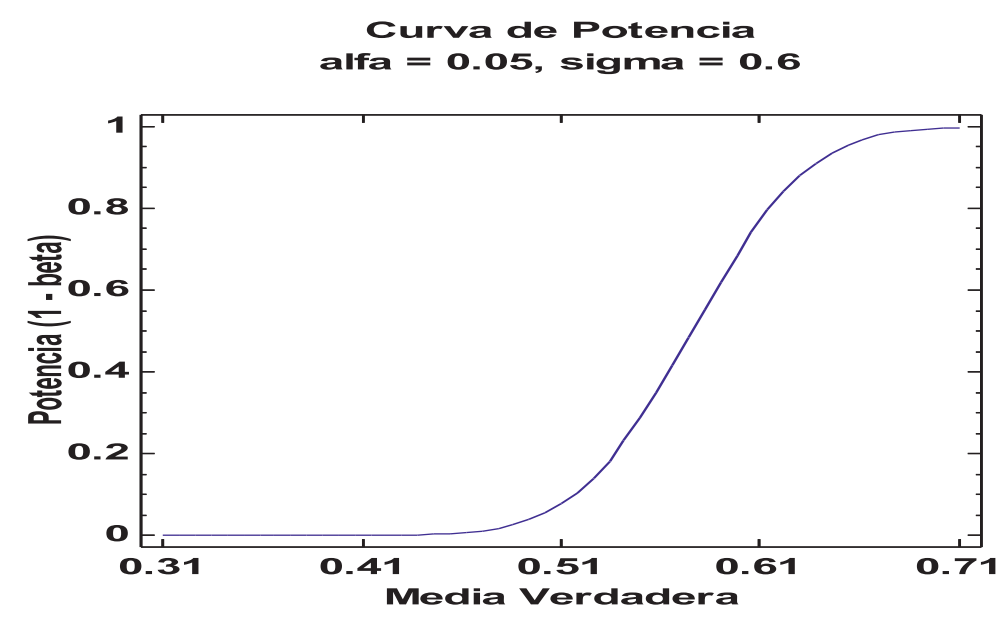




\section{DISCUSIÓN}

Los análisis presentados nos muestran que el nivel de control en las compras cuyos montos son iguales o menores a ocho es bajo; el nivel de eficiencia que alcanza las compras directas en la Zona Registral $N^{\circ}$ XIII también es bajo; y, el nivel de control incide significativamente en la eficiencia de compras directas en la Zona Registral $N^{\circ}$ XIII - Sede Tacna. 2016. Estos resultados han coincidido con la investigación de Mauricio, G. (2013) que señala que las reformas de la contabilidad gubernamental en Colombia y en Perú, son innovaciones en gobernanza. Pese a que existen diferentes estímulos, la reforma del Estado, la doctrina dominante y el papel de los organismos e instituciones financieras multilaterales, se tornan determinantes para introducir las agendas de las reformas. Asimismo, están relacionados con la investigación de: Paima, B., (2013). Influencia del sistema de control interno del área de compras en la rentabilidad de la empresa Autonort Trujillo S.A de la ciudad de Trujillo. (Universidad Privada Antenor Orrego-Trujillo).

Las entidades al no contar con ninguna normativa que regule el procedimiento para las compras directas por montos iguales o menores a ocho unidades impositivas tributarias (8) UIT-s, estas carecen de supervisión por parte del Órgano de Control Institucional $(\mathrm{OCl})$, dado que no existe ningún procedimiento que ellos puedan aplicar. Al no existir normativa, la entidad emite su propia directiva de acuerdo a sus necesidades, la cual no es muy estricta a comparación de la que podría emitir el OSCE. Las entidades al no contar con ninguna normativa que regule el procedimiento para las compras directas por montos iguales o menores a ocho unidades impositivas tributarias (8) UIT-s, no pueden evaluar los riesgos que tienen estas contrataciones, incidiendo en el nivel de eficiencia en las compras directas. El Organismo Supervisor de las Contrataciones del Estado (OSCE), debe elaborar una directiva general sobre el total procedimiento de las contrataciones directas de bienes y servicios por montos iguales o menores a ocho unidades impositivas tributarias (8) UIT-s, con la finalidad de uniformizar criterios de procedimientos a todas las entidades que se rigen por la Ley de Contrataciones del Estado. En la citada Directiva debe incluirse penalidades y obligaciones de ambas partes (entidad - proveedor), considerando el monto de las 8 UITs y así los Órganos de Control Institucional (OCI), deben de evaluar los riesgos a las contrataciones directas por montos iguales o menores a ocho unidades impositivas tributarias (8) UIT-s, con la finalidad de que las entidades puedan adquirir bienes y servicios con eficiencia.

Presentado: Marzo 2017

Aceptado: Junio 2017

\section{REFERENCIAS BIBLIOGRÁFICAS}

Avilés, L. G. (2007). Análisis de la corrupción en la adquisición y contratación del estado peruano. Universidad San Martín de Porres.

Caballero, L. (2011). Manuales de contrataciones. 6566. Lima: Caballero Bustamante.

Caroy, M. (2010). Análisis del efecto en la generación del empleo, de la política de fomento de la pequeña empresa a través de las compras estatales. Universidad Católica del Perú.

Contraloría General de la República. (1998). Técnicas de Control Interno para el Sector Público. Lima.

Cruz, C. O. (2011). Diseño de un sistema de control interno para contrataciones del Estado en el Hospital "La Caleta" de Chimbote, Perú.

Gómez, M. (2013). La reforma de la gestión pública en latinoamérica: Su impaacto en la transparencia y divulgación de la información financiera. Valencia, España: Universidad de Valencia.

Mantilla, S. (2005). Control inteeno - Método COSO. Lima.

Organismo Supervisor de las Contrataciones del Estado. (2013). Ley de Contrataciones del
Estado y su Reglamento. Rios S.A.C.

Paima, B., \& Villalobos, M. (2013). Influencia del sistema de control interno del área de compras en la rentabilidad de la empresa Autonort Trujillo S.A. de la Ciudad de Trujillo. Trujillo: Universidad Privada Antenor Orrego.

Singaucho, T. (2010). Control interno. 45-49. Lima.

Suárez, R. (2011). Modelado del sistema de apoyo a las modalidades de contratación pública según lo establecido en la legislación nacional: caso defensa pública. Venezula: Univesidad Católica Andrés Bello.

Torricelli Farfan, L. O. (2006). Influencia del curso de capacitación para el conocimiento de la nueva ley de contrataciones y adquisiciones del estado, dirigido a los empleados del Consejo Superior de Contrataciones y Adquisiciones del Estado (CONSUCODE) en el año 2005. Lima.

Zarpan Effio, Olivia Rosario .municipalidad Distrital De Pomalca. POMALCA, 30 DE MAYO DEL 2016. Perú.

Schultz, Carl. " Decisions marking in standbay services systems". 2000. 